\title{
Pitavastatin maintains MAPK7 expression and alleviates angiotensin II-induced vascular endothelial cell inflammation and injury
}

\author{
MIN LI ${ }^{1}$ and XIAOHUA LIU ${ }^{2}$ \\ ${ }^{1}$ Department of Outpatient Nursing, The Second Affiliated Hospital of Zhejiang University School of Medicine, \\ Hangzhou, Zhejiang 310000; ${ }^{2}$ Department of Nursing, Chengdu Shuangliu District Maternal and \\ Child Health Hospital, Chengdu, Sichuan 610200, P.R. China
}

Received August 9, 2021; Accepted October 25, 2021

DOI: $10.3892 /$ etm.2021.11055

\begin{abstract}
Statins have been reported to suppress vascular remodeling in rats with spontaneous hypertension. However, the possible effects of the statin pitavastatin on hypertension-induced endothelial inflammation and injury remain to be fully elucidated. The present study aimed to evaluate the effects of pitavastatin on HUVEC injury and inflammation. HUVECs were exposed to angiotensin (Ang) II with or without pitavastatin co-treatment, after which MAPK7 expression was detected via reverse transcription-quantitative PCR and western blotting. MAPK7 expression was additionally silenced in HUVECs via transfection with short hairpin RNA, followed by Ang II treatment with or without pitavastatin. Cell viability, inflammation, reactive oxygen species (ROS) production, nitric oxide (NO) production and cell apoptosis were then measured by using Cell Conting Kit-8, ELISA, commercial corresponding kits and TUNEL staining, respectively. Western blotting was also used to determine the protein expression of endothelial NO synthase and endothelin 1 , and the proteins involved in apoptosis. Results of the present study demonstrated that the expression levels of MAPK7 in Ang II-induced endothelial cells were decreased, which was reversed following treatment with pitavastatin. Pitavastatin reversed the Ang II-induced reduction in cell viability and reversed the Ang II-induced increase in inflammatory factor and ROS levels and apoptosis in HUVECs by activating MAPK7. Treatment with pitavastatin also increased the production of NO in addition to increasing the expression of endothelial NO synthase and endothelin-1 in Ang II-induced HUVECs through MAPK7 activation. Collectively, results
\end{abstract}

Correspondence to: Dr Xiaohua Liu, Department of Nursing, Chengdu Shuangliu District Maternal and Child Health Hospital, 396 Dongsheng Street, Chengdu, Sichuan 610200, P.R. China E-mail: liuxiaohuahh@126.com

Key words: pitavastatin, MAPK7, vascular endothelial, cell inflammation, cell injury from the present study demonstrated that treatment with pitavastatin preserves MAPK7 expression to alleviate Ang II-induced vascular endothelial cell inflammation and injury. Therefore, findings of the present study may help to elucidate the mechanisms underlying the effects of pitavastatin on vascular endothelial cell inflammation and injury.

\section{Introduction}

Hypertension is associated with high rates of incidence worldwide, which is considered to be major risk factor for stroke, coronary heart disease and chronic kidney disease (1). A previous study determined that $\sim 1$ billion people had primary hypertension, worldwide (2). Selection of treatment for hypertension remains to be a complex medical issue, since patients may suffer with side effects associated with the medication used to treat hypertension (3). Although an increasing number of pathological factors have been associated with hypertension, the specific mechanism of its pathogenesis remains unclear. Results of previous studies have demonstrated that endothelial dysfunction is closely associated with the occurrence and development of hypertension $(4,5)$. Endothelial dysfunction is considered to be one of the main causes of microvascular and macrovascular complications associated with hypertension (4,5). In addition, endothelial dysfunction has been reported to be a major factor underlying hypertension in the heart and kidneys, causing organ dysfunction (6). Endothelial dysfunction is characterized by changes in permeability and impaired vasodilation, which have been closely associated with both physiological and pathological processes, including aging, apoptosis, nitric oxide (NO) production and autophagy (7). Results of previous studies have demonstrated that high blood pressure can affect the function of endothelial cells through a number of methods $(8,9)$. High blood pressure has been reported to suppress endothelial-dependent vasodilation by NO, increase the permeability of endothelial cells, induce endothelial cell oxidative stress, promote endothelin 1 (ET-1) and angiotensin II (Ang II) production and promote inflammation (10). In turn, these may lead to endothelial cell dysfunction to aggravate hypertension and damage the organ involved $(11,12)$. Endothelial function is a 
key prognostic indicator of hypertension, where endothelial dysfunction limits the auto-repair and regeneration function of endothelial cells (13). Therefore, it remains of key importance to explore novel targets of endothelial cells to protect against endothelial cell injury and to more effectively diagnose and treat hypertension.

ERK5 is also known as MAPK7 and is a key signaling molecule in the maintenance of endothelial cell integrity and homeostasis (14). Atorvastatin, a type of statin, can be used for the treatment of hypertension (15). In rat models of spontaneous hypertension, atorvastatin has been documented to reduce vascular remodeling by activating protein kinase D/ERK5 (16,17). Pitavastatin is an alternative statin that is excreted in the feces through the hepatoenteric circulation $(13,14)$. Only a small portion of pitvastatin is selectively absorbed by hepatocytes and metabolized by the cytochrome system, meaning that it can avoid interactions with other drugs metabolized by this enzyme system $(18,19)$. However, the effects of pitavastatin in endothelial cell inflammation and injury induced by hypertension remain to be elucidated.

Therefore, the aim of the present study was to investigate the mechanism underlying the effects of pitavastatin on endothelial cell physiology and its potential association with ERK5.

\section{Materials and methods}

Materials. Ang II was purchased from APExBIO Technology LLC, whilst pitavastatin was purchased from HL Genomics, Co., Ltd. Antibodies were purchased as follows: Anti-phosphorylated (p)-endothelial NO synthase (eNOS; cat. no. ab215717; 1:1,000; Abcam), anti-eNOS (cat. no. ab76198; 1:1,000; Abcam), anti-endothelin (ET)1 (cat. no. ab2786; 1:1,000; Abcam), anti-Bcl-2 (cat. no. ab32124; 1:1,000; Abcam), anti-Bax (cat. no. ab32503; 1:1,000; Abcam), anti-cleaved caspase-3 (cat. no. ab2302; 1:1,000; Abcam), anti-cleaved poly (ADP-ribose) polymerase 1 (PARP1; cat. no. ab32064; 1:1,000; Abcam), anti-ERK5 (cat. no. ab40809; 1:1,000; Abcam), anti-GAPDH (cat. no. ab181602; 1:2,000; Abcam), HRP-conjugated goat anti-mouse IgG H\&L (cat. no. ab205719; 1:10,000; Abcam), HRP-conjugated goat anti-rabbit IgG H\&L (cat. no. ab205718; 1:10,000; Abcam) and Alexa Fluor ${ }^{\circledR} 488$ goat anti-rabbit IgG H\&L (cat. no. ab150077; 1:10,000; Abcam).

The following kits were purchased as follows: NO Colorimetric assay kit (cat. no. E-BC-K035-S; Elabscience Biotechnology, Inc.), reactive oxygen species (ROS) colorimetric assay kit (cat. no. E-BC-K138-F; Elabscience Biotechnology, Inc.), TNF- $\alpha$ ELISA kit (cat. no. ADI-901-099; Enzo Life Sciences, Inc.), IL-1 $\beta$ ELISA kit (cat. no. EHC002b.48; NeoBioscience Technology Co., Ltd.) and the IL-6 ELISA kit (cat. no. ADI-901-033; Enzo Life Sciences, Inc.).

Bioinformatics analysis. To search for the potential targets of pitavastatin, the online database STITCH (https://stitch.embl. $\mathrm{de} /$ ) was used by entering 'pitavastatin' in the 'Item Name' box. The minimum required interaction score was set to $>0.4$.

Cell lines and transfection. HUVECs (cat. no. BNCC347734; an extensively used endothelial cell model in vitro) (20) and vascular cell basal medium (cat. no. BNCC341789) containing $5 \%$ FBS, were purchased from BeNa Culture Collection; Beijing Beina Chunglian Biotechnology Research Institute. HUVECs were cultured in $5 \% \mathrm{CO}_{2}$ with $95 \%$ humidity at $37^{\circ} \mathrm{C}$ in a $\mathrm{CO}_{2}$ incubator.

To construct the lentivirus vector expressing MAPK7 short hairpin (sh)-RNA (shRNA-MAPK7-1 or shRNA-MAPK7-2), shRNA sequences were annealed and inserted between the $E c o$ RI and $B g l \mathrm{II}$ sites of the pHBLV-U6-Puro vector (Hanbio Biotechnology Co., Ltd.). The vectors were then co-transfected along with the packaging plasmids including Gal-pol, Rev and VSV-G (all from Yunzhou Bio, China) in a ratio of 2:1:1:1, into 293T cells (ATCC) using Lipofectamine ${ }^{\circledR} 2000$ (Thermo Fisher Scientific, Inc.). After incubation at $37^{\circ} \mathrm{C}$ for $72 \mathrm{~h}$, the culture medium was collected and centrifuged at $500 \mathrm{x} \mathrm{g}$ for $5 \mathrm{~min}$ at room temperature to obtain the viral supernatant. Finally, HUVECs were transfected with the lentiviral shRNAs ( $2^{\text {rd }}$ generation) at a MOI of $\sim 3$ and incubated at $37^{\circ} \mathrm{C}$. Puromycin (Thermo Fisher Scientific, Inc.) selection $(2 \mu \mathrm{g} / \mathrm{ml})$ was added 2 days post-transfection and after another 2 days incubation at $37^{\circ} \mathrm{C}$, transfection efficiency was detected using western blot analysis. The shRNA sequences were listed as follows: shRNA-MAPK7-1 forward 5'-CCGGACAAGTTA CTAAGCCATATTTCTCGAGAAATATGGCTTAGTAAC TTG TTTTTTG-3' and reverse, 5'-AATTCAAAAAACAAG TTACTAAGCCATATTTCTCGAGAAATATGGCTTAGTAA CTTGT-3'; shRNA-MAPK7-2 forward, 5'-CCGGTTGTT AGGAGACAAGTTAAATCTCGAGATTTAACTTGTCTC CTAACAATTTTTG-3' and reverse, 5'-AATTCAAAAATT GTTAGGAGACAAGTTAAATCTCGAGATTTAACTTGT CTCCTAACAA-3'.

Cell viability assay. Cell Counting Kit-8 (CCK-8; cat. no. C0039; Beyotime Institute of Biotechnology) assays were performed to measure cell viability. HUVECs were inoculated into 96 -well plates $\left(2 \times 10^{3}\right.$ cells/well). After being treated with $1 \mu \mathrm{M}$ Ang II with or without $1 \mu \mathrm{M}$ pitavastatin co-treatment for $24 \mathrm{~h}$ at $37^{\circ} \mathrm{C}$, cells were incubated with $20 \mu \mathrm{l}$ CCK-8 solution per well at $37^{\circ} \mathrm{C}$ for $2 \mathrm{~h}$. The absorbance was measured at $450 \mathrm{~nm}$ using a spectrophotometer.

TUNEL assay. TUNEL staining was performed using the ApopTag Fluorescein in situ Apoptosis Detection kit (cat. no. S7110; Sigma-Aldrich; Merck KGaA) following the manufacturer's protocol. Briefly, after being treated with $1 \mu \mathrm{M}$ Ang II with or without $1 \mu \mathrm{M}$ pitavastatin co-treatment at $37^{\circ} \mathrm{C}$ for $24 \mathrm{~h}$, control (untransfected) or transfected HUVECs ( $\left.2 \times 10^{4}\right)$ were washed with PBS and subsequently fixed using $4 \%$ paraformaldehyde for $30 \mathrm{~min}$ at room temperature. Cells were incubated for $90 \mathrm{~min}$ at $37^{\circ} \mathrm{C}$ with the terminal deoxynucleotidyltransferase (TdT) incubation buffer. The negative control slide was incubated without the TdT enzyme. The reaction was terminated by washing with PBS and the slide was examined under a fluorescence microscope (Nikon Eclipse 80i; Nikon Corporation). DAPI $(5 \mu \mathrm{g} / \mathrm{ml})$ was used to stain the nuclei blue at room temperature for $5 \mathrm{~min}$. Three fields of view per well were observed (magnification, $\mathrm{x} 200$ ) and the percentage of TUNEL positive cells was calculated using ImageJ software (version 1.8.0; National Institutes of Health) as follows: TUNEL-positive cells $(\%)=$ the density of green/blue $\times 100 \%$. 
A

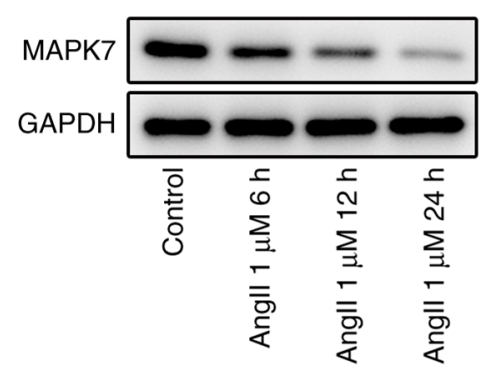

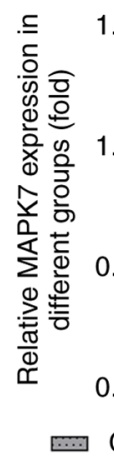

B

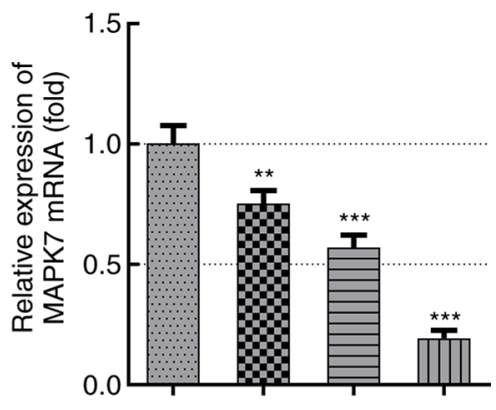

C

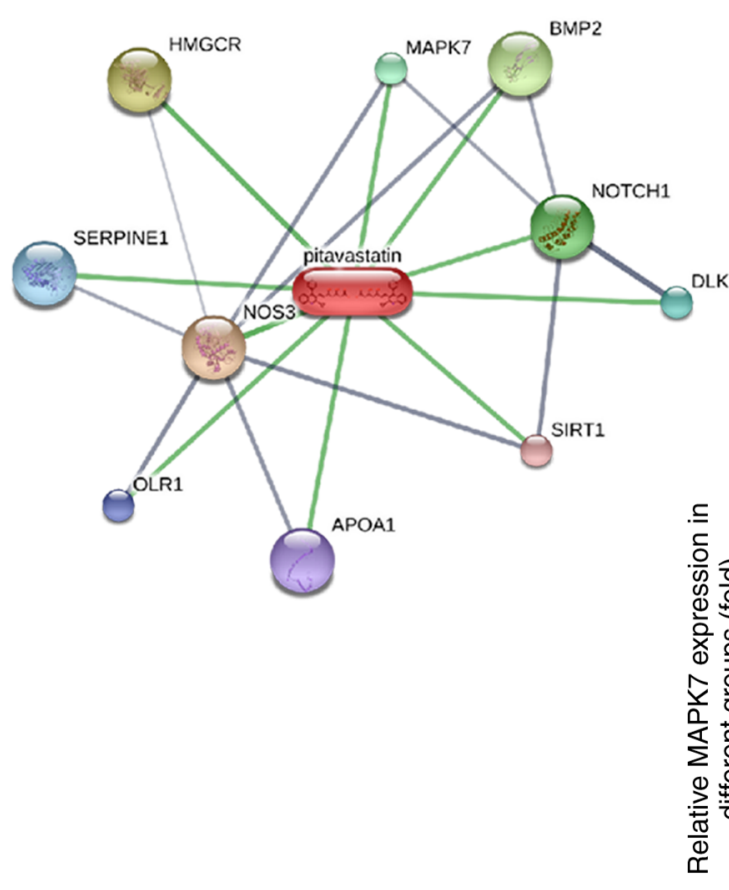

D
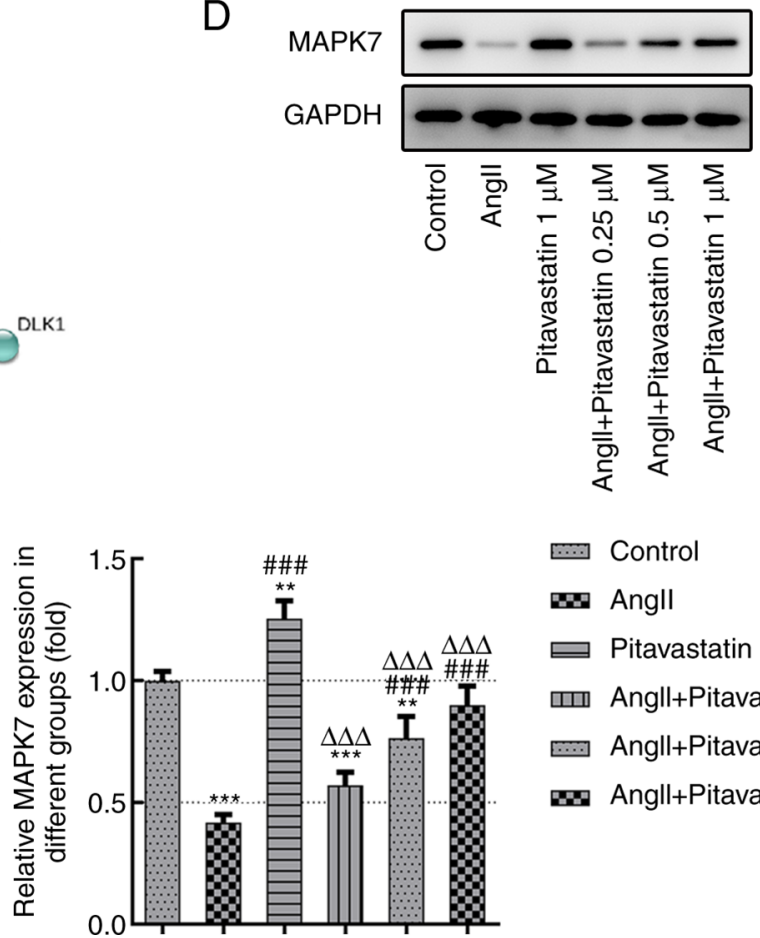

…. Control

Angll

$\sqsupseteq$ Pitavastatin $1 \mu \mathrm{M}$

س] Angll+Pitavastatin $0.25 \mu \mathrm{M}$

Angll+Pitavastatin $0.5 \mu \mathrm{M}$

W Angll+Pitavastatin $1 \mu \mathrm{M}$

Figure 1. Pitavastatin increases MAPK7 expression in Ang II-induced endothelial cells. Expression levels of MAPK7 in Ang II-induced endothelial cells were measured using (A) western blot analysis and (B) reverse transcription-quantitative PCR. (C) STITCH predicted that pitavastatin targeted MAPK7 expression. (D) Expression of MAPK7 was detected using western blot analysis. ${ }^{*} \mathrm{P}<0.05,{ }^{* *} \mathrm{P}<0.01$ and ${ }^{* * *} \mathrm{P}<0.001$ vs. Control; ${ }^{\# \# \#} \mathrm{P}<0.001$ vs. Ang II; ${ }^{\Delta \Delta \Delta} \mathrm{P}<0.001$ vs. $1 \mu \mathrm{M}$ pitavastatin. Ang II, angiotensin II.

Detection of NO and ROS. HUVECs were inoculated into 96-well plates $\left(2 \times 10^{3}\right.$ cells/well). After treatment with $1 \mu \mathrm{M}$ Ang II with or without $1 \mu \mathrm{M}$ pitavastatin co-treatment for $24 \mathrm{~h}$ at $37^{\circ} \mathrm{C}$, detection of $\mathrm{NO}$ and ROS levels in the HUVEC culture medium was performed using the aforementioned commercial kits. Levels of NO and ROS were measured at $520 \mathrm{~nm}$ using a Multiskan Mk3 microplate reader (Thermo Fisher Scientific, Inc.).

ELISA. Concentrations of TNF- $\alpha$, IL- $1 \beta$ and IL- 6 in the HUVEC culture medium were detected using the aforementioned ELISA kits according to the manufacturer's protocol. Expression levels were quantified using a Multiskan Mk3 microplate reader (Thermo Fisher Scientific, Inc.).

Reverse transcription-quantitative PCR (RT-qPCR). Total RNA from HUVECs was collected using the TRIzol agent (Thermo Fisher Scientific, Inc.) according to the manufacturer's protocol, then reversely transcribed into cDNA using
PrimeScript ${ }^{\mathrm{TM}}$ RT Master Mix (cat. no. RR036A; Takara Bio, Inc.) according to the manufacturer's protocol. Subsequently, qPCR was performed using TB Green ${ }^{\mathrm{TM}}$ Fast qPCR Mix (Takara Bio, Inc.) on an Applied Biosystems 7500 Real-Time PCR System (Thermo Fisher Scientific, Inc.). The results were analyzed using the $2^{-\Delta \Delta \mathrm{Cq}}$ method (21). The thermocycling conditions used for qPCR were: $95^{\circ} \mathrm{C}$ for $2 \mathrm{~min}$, followed by 40 cycles of $95^{\circ} \mathrm{C}$ for $20 \mathrm{sec}$ and $65^{\circ} \mathrm{C}$ for $40 \mathrm{sec}$. The sequences of the primers were as follows: MAPK7 forward, 5'-ACACGA CAACATCATCGCCA-3' and reverse, 5'-TCCAGGACCACG TAGACAGA-3' and $\beta$-actin forward, 5'-ACAGAGCCTCGC CTTTGCC-3' and reverse, 5'-GATATCATCATCCATGGT GAGCTGG-3'. $\beta$-actin was used as the internal reference.

Western blot analysis. Total protein was extracted from the cell lysate using the RIPA buffer (Beyotime Institute of Biotechnology). The protein samples were quantified using a BCA assay (Pierce; Thermo Fisher Scientific, Inc.). Subsequently, extracted proteins (50 $\mu \mathrm{g}$ per lane) were 
A

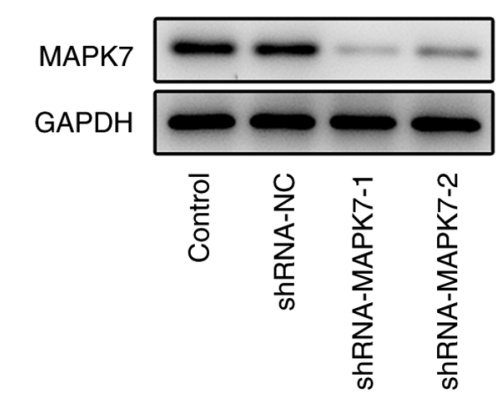

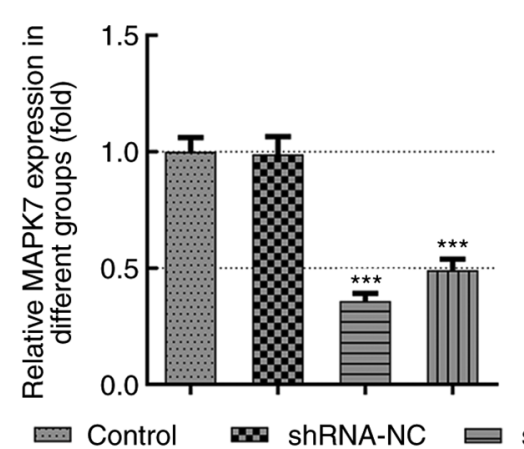

B

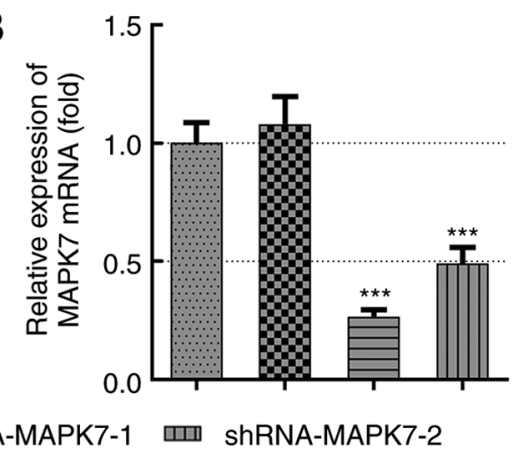

C

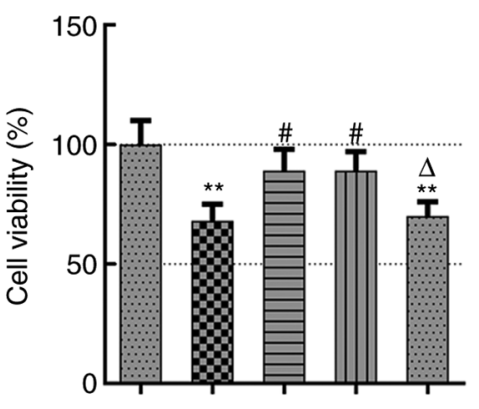

… Control

Angll

曰Angll+Pitavastatin $1 \mu \mathrm{M}$

سلس Angll+Pitavastatin $1 \mu \mathrm{M}+$ shRNA-NC

Angll+Pitavastatin $1 \mu \mathrm{M}+$ shRNA-MAPK7

D
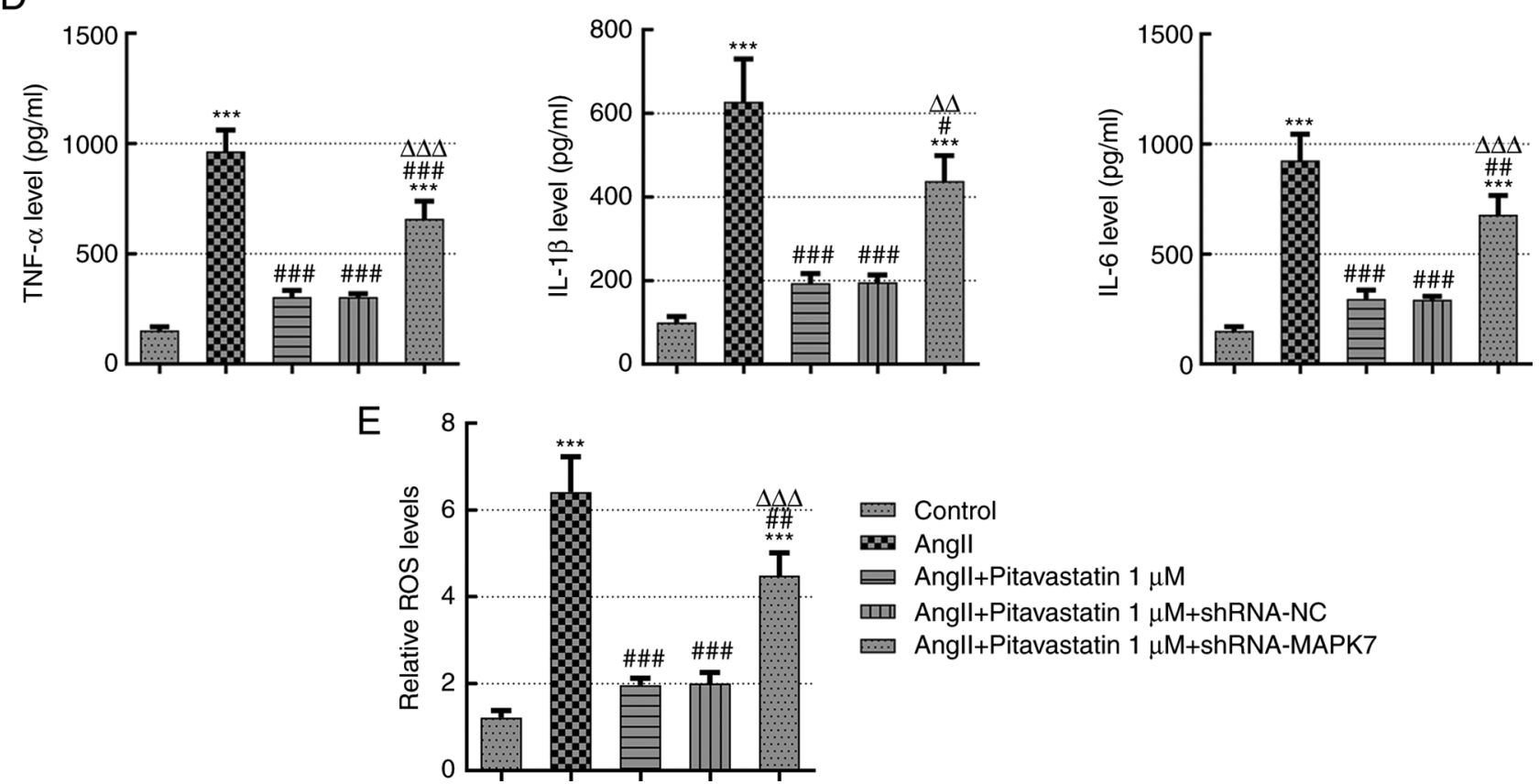

Figure 2. Pitavastatin alleviates damage in Ang II-induced HUVECs by activating MAPK7. MAPK7 expression in HUVECs was measured using (A) western blot analysis and (B) reverse transcription-quantitative PCR. ${ }^{* * *} \mathrm{P}<0.001$ vs. shRNA-NC. (C) Cell Counting Kit- 8 assay was used to measure the cell viability of Ang II-induced HUVECs following treatment with different concentrations of pitavastatin. (D) ELISA were used to measure the production of TNF- $\alpha$, IL-1 $\beta$ and IL-6. (E) Commercial kits were used to detect the levels of reactive oxygen species. ${ }^{* *} \mathrm{P}<0.01$ and ${ }^{* * * *} \mathrm{P}<0.001$ vs. Control; ${ }^{\#} \mathrm{P}<0.05,{ }^{\# \#} \mathrm{P}<0.01$ and

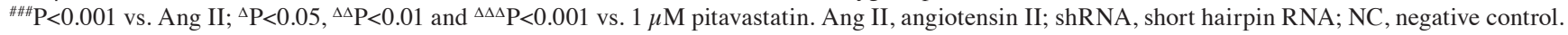

separated by $10 \%$ SDS-PAGE and transferred onto PVDF membranes (EMD Millipore). Membranes were blocked with $5 \%$ skimmed milk at room temperature for $2 \mathrm{~h}$ and subsequently incubated with the aforementioned primary antibodies overnight at $4^{\circ} \mathrm{C}$. Following primary antibody incubation, membranes were incubated with the aforementioned corresponding secondary antibodies at room temperature for $2 \mathrm{~h}$. Protein bands were visualized using Pierce ${ }^{\mathrm{TM}}$ ECL (Thermo Fisher Scientific, Inc.). Protein expression was quantified using ImageJ software (version 1.8.0; National Institutes of Health).
Statistical analysis. All experiments were performed in triplicate. All data are presented as the mean \pm standard deviation and samples were evaluated using one-way ANOVA followed by Tukey's test using SPSS 16.0 (SPSS, Inc.). An unpaired Student's $\mathrm{t}$ tests were used for comparisons between two groups. $\mathrm{P}<0.05$ was considered to indicate a statistically significant difference.

\section{Results}

Pitavastatin increases MAPK7 expression in Ang II-induced endothelial cells. As shown in Fig. 1A and B, the expression 
A

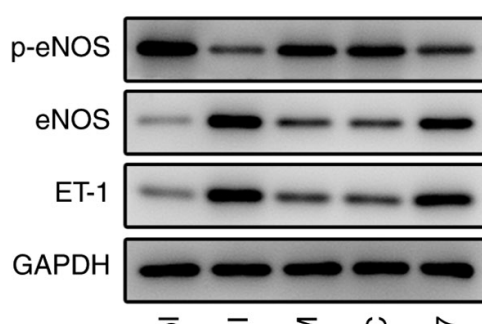

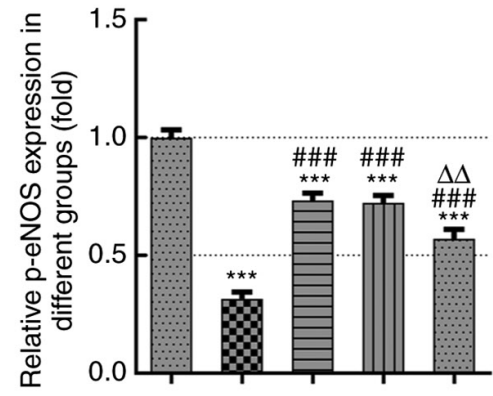

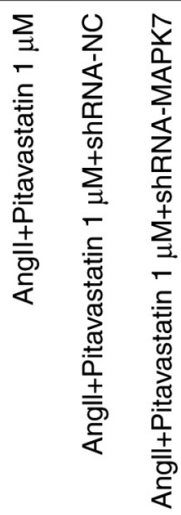

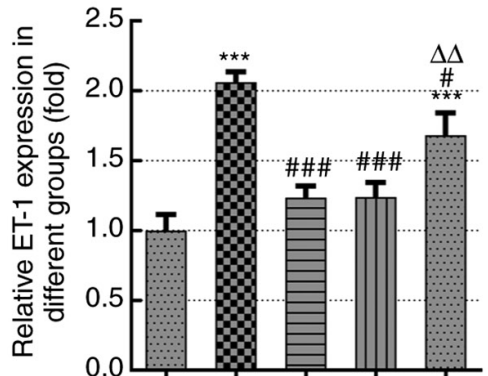

B
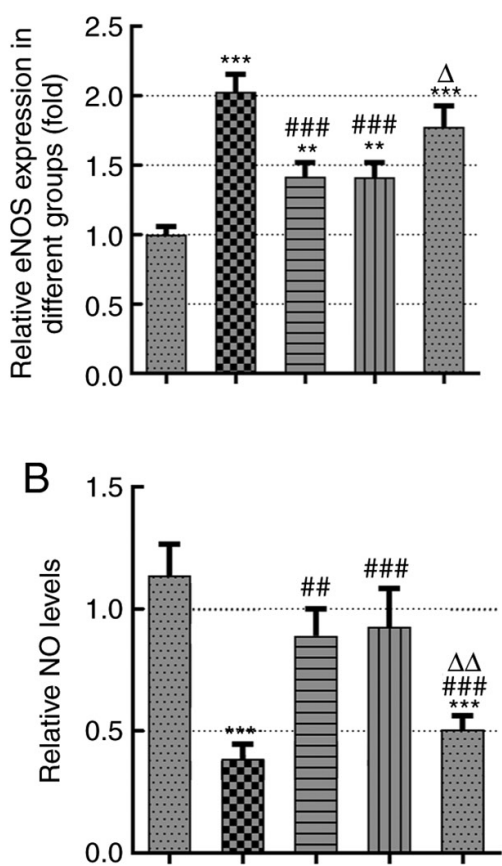

Figure 3. Pitavastatin increases the production of NO, activation eNOS and ET-1 expression in Ang II-induced HUVECs by activating MAPK7. (A) Protein expression of eNOS phosphorylation, eNOS expression and ET-1 expression in Ang II-induced endothelial cells were measured using western blot analysis. (B) NO production was measured using a commercial kit. ${ }^{* *} \mathrm{P}<0.01$ and ${ }^{* * *} \mathrm{P}<0.001$ vs. Control; ${ }^{\#} \mathrm{P}<0.05,{ }^{\# \#} \mathrm{P}<0.01$ and ${ }^{\# \# \#} \mathrm{P}<0.001$ vs. Ang II; ${ }^{\Delta} \mathrm{P}<0.05$ and ${ }^{\Delta} \mathrm{P}<0.01$ vs. Ang II $+1 \mu \mathrm{M}$ pitavastatin. Ang II, angiotensin II; shRNA, short hairpin RNA; NC, negative control; ET-1, endothelin-1; NO, nitric oxide; p-, phosphorylated; eNOS, endothelial NO synthase.

levels of MAPK7 were detected using RT-qPCR and western blot analyzes. The results demonstrated that the expression levels of MAPK7 were significantly decreased following treatment with $1 \mu \mathrm{M}$ Ang II in a time-dependent manner. In particular, MAPK7 expression was the lowest following treatment with $1 \mu \mathrm{M}$ Ang II for $24 \mathrm{~h}$ (Fig. 1A and B). Therefore, this treatment time was selected for subsequent studies. The STITCH (http://stitch.embl.de/) database was subsequently used to predict potential pitavastatin targets, which revealed MAPK7 as one of the hits (Fig. 1C). Following pitavastatin treatment, the expression levels of MAPK7 were detected using western blot analysis. Results of the present study demonstrated that the expression levels of MAPK7 in Ang II-induced HUVECs were increased following treatment with pitavastatin, especially at higher doses of 0.5 and $1 \mu \mathrm{M}$ (Fig. 1D). In conclusion, pitavastatin restored MAPK7 expression in Ang II-induced endothelial cells.

Pitavastatin alleviates damage in Ang II-induced HUVECs by activating MAPK7. To determine the effects of pitavastatin on Ang II-induced endothelial cell viability and inflammation, loss of function experiments were performed. HUVECs were transfected with shRNA-NC, shRNA-MAPK7-1 or shRNA-MAPK7-2 for MAPK7 silencing. As demonstrated in Fig. 2A and B, there was no significant difference in MAPK7 expression between the sh-negative control (NC) group compared with that in the control group. However, MAPK7 expression levels were significantly reduced in HUVECs transfected with sh-MAPK7-1 or sh-MAPK7-2 compared with those in the sh-NC group (Fig. 2A and B). Since the transfection efficiency was the highest following transfection with sh-MAPK7-1, this shRNA was used for subsequent experiments.

In addition, cell viability were significantly decreased following treatment with Ang II, which was significantly reversed by pitavastatin treatment (Fig. 2C). By contrast, this pitavastatin-induced restoration of cell viability was significantly prevented by MAPK7 knockdown (Fig. 2C). As demonstrated in Fig. 2D, the secretion of IL-1 $\beta$, IL-6 and IL-8 were significantly increased following treatment with Ang II but were significantly reversed by pitavastatin. The anti-inflammatory effects of pitavastatin on IL-1 $\beta$, IL- 6 and IL-8 were in turn significantly reversed by MAPK7 knockdown (Fig. 2D). In addition, results of the ROS kit demonstrated that the levels of ROS were significantly increased following Ang II treatment but were reversed by concomitant pitavastatin treatment, which was in turn reversed by transfection with shRNA-MAPK7 (Fig. 2E).

Pitavastatin increases the production of NO, eNOS phosphorylation and ET-1 expression in Ang II-induced HUVECs by activating MAPK7. NO is a key signaling molecule secreted by the vascular endothelium that serves a key role in vascular smooth muscle relaxation by activating certain signal transduction pathways, such as that of hypoxiainducible factor-1 $\alpha$ and VEGF (22). eNOS is a key enzyme in the NO production pathway that is an indirect indicator of heart failure (23). By contrast, ET-1 is mainly secreted by 
A
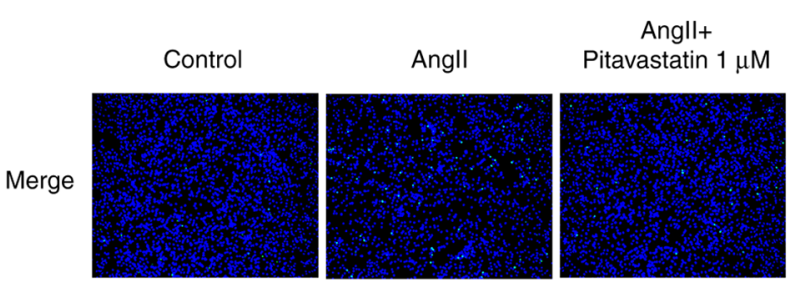
Pitavastatin $1 \mu \mathrm{M}+$ Pitavastatin $1 \mu \mathrm{M}+$ ShRNA-NC ShRNA-MAPK7
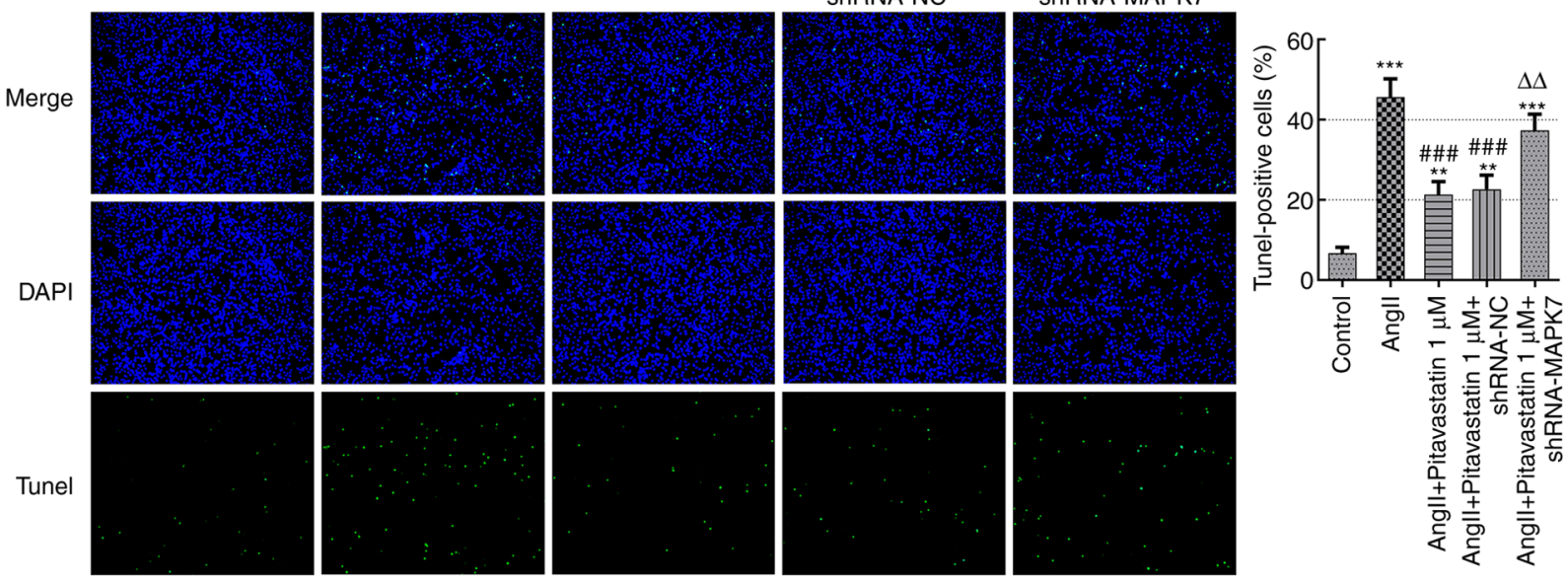

B
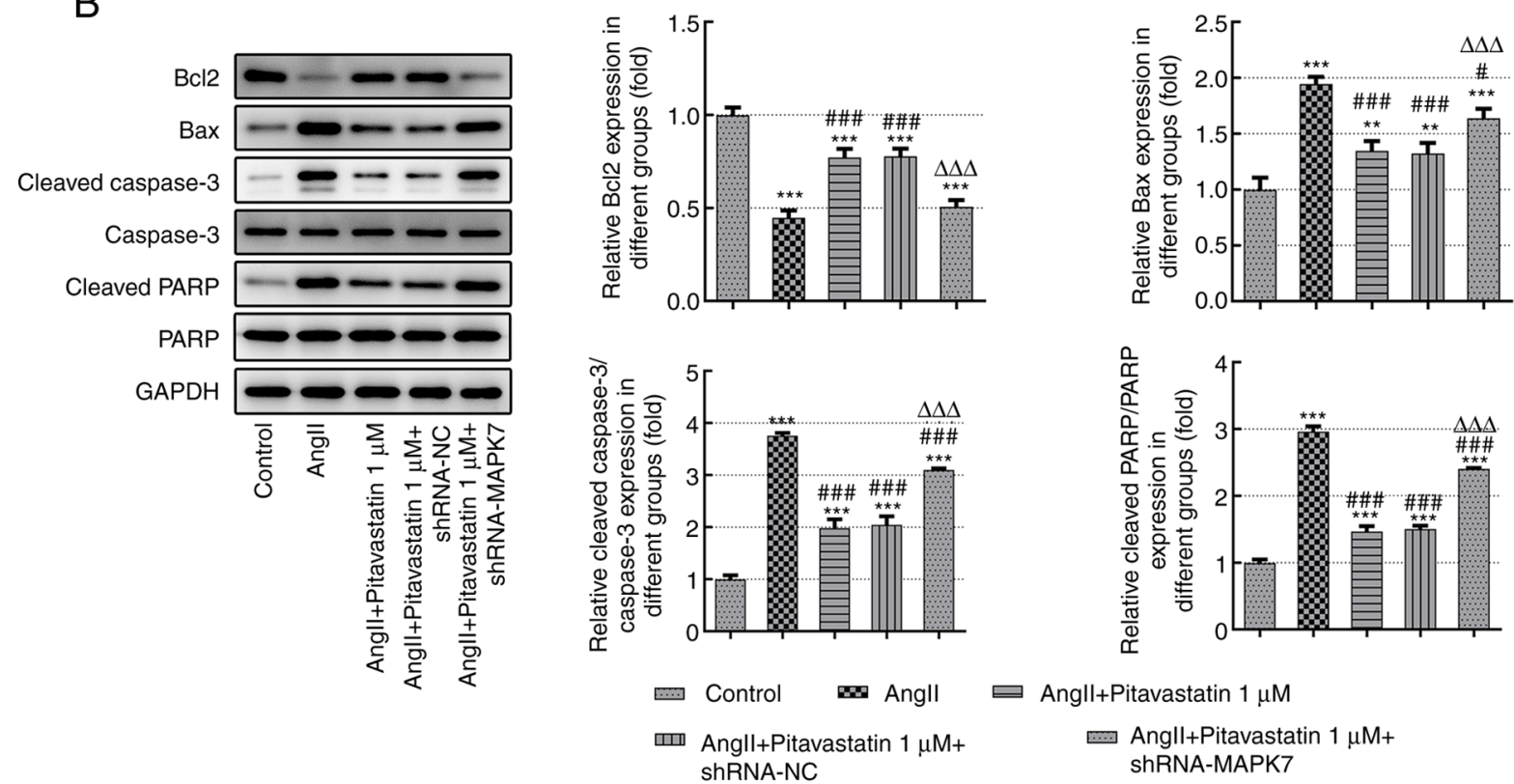

$$
\begin{aligned}
& \text { Control Angll } \square \text { Angll+Pitavastatin } 1 \mu \mathrm{M} \\
& \begin{array}{l}
\text { Angll+Pitavastatin } 1 \mu \mathrm{M}+ \\
\text { shRNA-NC }
\end{array}
\end{aligned}
$$

Figure 4. Pitavastatin inhibits the apoptosis of Ang II-induced HUVECs by activating MAPK7. (A) TUNEL staining was performed to investigate the apoptosis of Ang II-induced HUVECs at $24 \mathrm{~h}$ following treatment with pitavastatin. (B) Western blot analyses of Bax, Bcl-2, cleaved-PARP/PARP and cleaved caspase-3/caspase-3 protein levels in Ang II-induced HUVECs. ${ }^{* *} \mathrm{P}<0.01$ and ${ }^{* * * *} \mathrm{P}<0.001$ vs. Control; ${ }^{\#} \mathrm{P}<0.05$ and ${ }^{\# \# \#} \mathrm{P}<0.001$ vs. Ang II; ${ }^{\Delta \Delta} \mathrm{P}<0.01$ and ${ }^{\Delta \Delta \Delta} \mathrm{P}<0.001$ vs. Ang II $+1 \mu \mathrm{M}$ pitavastatin. Ang II, angiotensin II; NC, negative control; shRNA, short hairpin RNA; PARP, poly (ADP-ribose) polymerase 1 .

vascular endothelial cells, cardiomyocytes and endocardial cells and is considered to be a potent vasoconstrictor (24). ET-1 co-operates with NO to regulate the vasoconstrictive and diastolic balance of the vascular endothelium (10). As demonstrated in Fig. 3A, treatment with pitavastatin significantly suppressed the expression levels of eNOS and ET-1 in Ang II-induced HUVECs, which were significantly reversed following transfection with the sh-MAPK7 plasmid. Notably, treatment with pitavastatin in Ang II-induced HUVECs increased the production levels of NO and p-eNOS, which were significantly reversed following transfection with sh-MAPK7 (Fig. 3B).

Pitavastatin inhibits the apoptosis of Ang II-induced HUVECs by activating MAPK7. To assess the effects of pitavastatin on the apoptosis of Ang II-induced HUVECs, TUNEL staining was performed. The number of TUNEL-positive cells was significantly reduced in the Ang II + pitavastatin group compared with that in the Ang II-alone group (green represents apoptotic cells). However, transfection with shRNA-MAPK7 significantly reversed the inhibitory effects of pitavastatin on cell apoptosis (Fig. 4A). These results were investigated further by measuring the expression levels of apoptosis-associated proteins. As demonstrated in Fig. 4B, the expression levels of Bax, ratios of cleaved-PARP/PARP and cleaved caspase-3/caspase-3 in the Ang II-induced HUVECs were significantly reduced following treatment with pitavastatin. However, expression levels of the anti-apoptotic protein Bcl-2 were significantly increased by pitavastatin (Fig. 4B). All of the effects mediated by pitavastatin on the expression of proteins associated with apoptosis were significantly reversed by shRNA-MAPK7 transfection (Fig. 4B). These findings suggest that treatment with pitavastatin inhibited the apoptosis of Ang II-induced HUVECs by activating MAPK7. 


\section{Discussion}

Vascular endothelial cell injury is considered to be one of the first events of atherosclerosis and is closely associated with the development of cardiovascular diseases, including coronary heart disease and hypertension (25). Results of a previous study demonstrated that ischemia injury, oxidative stress and oxidized low density lipoprotein can all induce endothelial cell injury and apoptosis (26). In addition, the expression levels of eNOS and ET-1 were significantly increased following the induction of vascular endothelial injury, cell destruction or increased membrane permeability, which were accompanied with reductions in the levels of NO and may reflect the degree of cell injury $(27,28)$. In the present study, HUVECs were treated with $1 \mu \mathrm{M}$ Ang II, which significantly increased proinflammatory cytokine and ROS production and ET-1 secretion, significantly decreased NO production whilst significantly increasing the rate of apoptosis. These results suggest that Ang II is directly involved in the process of vascular endothelial cell injury and apoptosis.

Statins are lipid-lowering drugs that are frequently applied in clinical practice (29). They have been previously reported to exert anti-inflammatory, anti-oxidation, vascular endothelial protective, plaque stabilizing and anti-platelet properties (30). Results of the present study demonstrated that treatment with pitavastatin significantly increased the production levels of NO and eNOS phosphorylation whilst significantly decreasing ET-1 expression in Ang II-induced HUVECs. Furthermore, treatment with pitavastatin alleviated cell damage and apoptosis of Ang II-induced HUVECs by activating MAPK7, as demonstrated by MAPK7 knockdown experiments.

MAPK is typically activated by epidermal growth factors, inflammatory factors and growth factors, which has been found to regulate the stress response, inflammatory response, cell proliferation and development $(31,32)$. Results of a previous study demonstrated that MAPK7 deficiency alleviated DNA damage in mouse thymus cells and inhibited the growth of mouse thymus lymphoma (33). Gavine et al (34) revealed that MAPK7 expression was higher in squamous cell carcinoma and esophageal carcinoma tissues compared with normal tissues, whilst MAPK7 silencing inhibited the proliferation, migration and invasion of osteosarcoma cells to enhance their sensitivity to chemotherapy. In the present study, treatment with pitavastatin increased MAPK7 expression in Ang II-induced endothelial cells. In addition, it is known that MAPK7 signaling is activated upon phosphorylation (35), therefore, the effects of pitavastatin on the phosphorylation of MAPK7 and the effects of an MAPK7 inhibitor on the effects of pitavastatin should be investigated in future studies.

To conclude, the present study provided supporting evidence that pitavastatin can preserve MAPK7 expression to alleviate Ang II-induced vascular endothelial cell inflammation and injury. Results of the present study revealed a potentially novel therapeutic strategy for the treatment of vascular endothelial cell inflammation and injury. However, the present study solely focused on the pitavastatin-induced regulation of endothelial cell proliferation, inflammation and apoptosis. Further investigations are required to focus on the role of associated signaling pathways.

\section{Acknowledgements}

Not applicable.

\section{Funding}

No funding was received.

\section{Availability of data and materials}

The datasets used and/or analyzed during the current study are available from the corresponding author on reasonable request.

\section{Author's contributions}

ML and XHL contributed to the conception and design of the study. ML and XHL performed the experiments and collected the data. ML and XHL performed the statistical analysis. ML and XHL completed data interpretation. Both authors contributed to reading and revising the manuscript and approved the submitted version. Both authors read and approved the final manuscript. ML and XHL confirm the authenticity of all the raw data.

\section{Ethics approval and consent to participate}

Not applicable.

\section{Patient consent for publication}

Not applicable.

\section{Competing interests}

The authors declare that they have no competing interests.

\section{References}

1. Di Palo KE and Barone NJ: Hypertension and heart failure: Prevention, targets, and treatment. Heart Fail Clin 16: 99-106, 2020.

2. Zhang W, Wang Q, Feng Y, Chen X, Yang L, Xu M, Wang X, Li W, Niu X and Gao D: MicroRNA-26a protects the heart against hypertension-induced myocardial fibrosis. J Am Heart Assoc 9: e017970, 2020.

3. Chen Y,Lu W, Yang K, Duan X, Li M, Chen X, Zhang J, Kuang M, Liu S, Wu X, et al: Tetramethylpyrazine: A promising drug for the treatment of pulmonary hypertension. Br J Pharmacol 177: 2743-2764, 2020

4. Zhao H, Wang Y, Zhang X, Guo Y and Wang X: miR-181b-5p inhibits endothelial-mesenchymal transition in monocrotaline-induced pulmonary arterial hypertension by targeting endocan and TGFBR1. Toxicol Appl Pharmacol 386: 114827, 2020.

5. Dong ZC, Wu MM, Zhang YL, Wang QS, Liang C, Yan X, Zou LX, Chen C, Han X, Zhang B and Zhang ZR: The vascular endothelial growth factor trap aflibercept induces vascular dysfunction and hypertension via attenuation of eNOS/NO signaling in mice. Acta Pharmacol Sin 42: 1437-1448, 2020.

6. Mokotedi L, Millen AME, Mogane C, Gomes M, Woodiwiss AJ, Norton GR and Michel FS: Associations of inflammatory markers and vascular cell adhesion molecule-1 with endothelial dysfunction in collagen-induced arthritis. Eur J Pharmacol 865: 172786, 2019.

7. Canpolat U, Kocyigit D and Yildirim A: Role of endothelial dysfunction and endocan in atherosclerosis: Point of origin or end point? Angiology 71: 477, 2020. 
8. Bhagwani AR, Hultman S, Farkas D, Moncayo R, Dandamudi K, Zadu AK, Cool CD and Farkas L: Endothelial cells are a source of nestin expression in pulmonary arterial hypertension. PLoS One 14: e0213890, 2019.

9. Luo S, Xia W, Chen C, Robinson EA and Tao J: Endothelial progenitor cells and hypertension: Current concepts and future implications. Clin Sci (Lond) 130: 2029-2042, 2016.

10. Masi S, Uliana M and Virdis A: Angiotensin II and vascular damage in hypertension: Role of oxidative stress and sympathetic activation. Vascul Pharmacol 115: 13-17, 2019.

11. Ballard KD, Timsina R and Timmerman KL: Influence of time of day and intermittent aerobic exercise on vascular endothelia function and plasma endothelin-1 in healthy adults. Chronobiol Int: 1-8, 2021.

12. Zhang X, Hu C, Yuan YP, Song P, Kong CY, Wu HM, Xu SC, Ma ZG and Tang QZ: Endothelial ERG alleviates cardiac fibrosis via blocking endothelin-1-dependent paracrine mechanism. Cel Biol Toxicol 37: 873-890, 2021.

13. Hu C and Dong ZL: MicroRNA-212 promotes the recovery function and vascular regeneration of endothelial progenitor cells in mice with ischemic stroke through inactivation of the notch signaling pathway via downregulating MMP9 expression. J Cell Physiol 234: 7090-7103, 2019.

14. Gentilini A, Lori G, Caligiuri A, Raggi C, Maira GD, Pastore M, Piombanti B, Lottini T, Arcangeli A, Madiai S, et al: Extracellular signal-regulated kinase 5 regulates the malignant phenotype of cholangiocarcinoma cells. Hepatology 74: 2007-2020, 2021.

15. Curran MP: Amlodipine/Atorvastatin: A review of its use in the treatment of hypertension and dyslipidaemia and the prevention of cardiovascular disease. Drugs 70: 191-213, 2010.

16. Fang T, Guo B, Xue L and Wang L: Atorvastatin prevents myocardial fibrosis in spontaneous hypertension via interleukin-6 (IL-6)/signal transducer and activator of transcription 3 (STAT3)/ endothelin-1 (ET-1) pathway. Med Sci Monit 25: 318-323, 2019.

17. Yuan H, Wang D, Zhang Y and Geng J: Atorvastatin attenuates vascular remodelling in spontaneously hypertensive rats via the protein kinase D/extracellular signal-regulated kinase 5 pathway. Clin Exp Pharmacol Physiol 47: 1429-1438, 2020.

18. Nagayama D, Saiki A, Watanabe Y, Yamaguchi T, Ohira M, Sato N, Kanayama M, Moroi M, Miyashita Y, Shirai K and Tatsuno I: Prevention of cardiovascular events with pitavastatin is associated with increased serum lipoprotein lipase mass level: Subgroup analysis of the TOHO-LIP. J Atheroscler Thromb: Feb 27, 2021. doi: 10.5551/jat.62141.

19. Bhatti H and Tadi P: Pitavastatin. In: StatPearls, StatPearls Publishing, Treasure Island, FL, 2021.

20. Cao Y, Gong Y, Liu L, Zhou Y, Fang X, Zhang C, Li Y and Li J: The use of human umbilical vein endothelial cells (HUVECs) as an in vitro model to assess the toxicity of nanoparticles to endothelium: A review. J Appl Toxicol 37: 1359-1369, 2017.

21. Livak KJ and Schmittgen TD: Analysis of relative gene expression data using real-time quantitative PCR and the 2(-Delta Delta C(T)) method. Methods 25: 402-408, 2001.

22. Rajendran S, Shen X, Glawe J, Kolluru GK and Kevil CG: Nitric oxide and hydrogen sulfide regulation of ischemic vascular growth and remodeling. Compr Physiol 9: 1213-1247, 2019.
23. Bonnefont-Rousselot D: Resveratrol and cardiovascular diseases. Nutrients 8: 250, 2016.

24. Jankowich M and Choudhary G: Endothelin-1 levels and cardiovascular events. Trends Cardiovasc Med 30: 1-8, 2020.

25. Yamada T, Egashira N, Imuta M, Yano T, Yamauchi $Y$, Watanabe $\mathrm{H}$ and Oishi R: Role of oxidative stress in vinorelbine-induced vascular endothelial cell injury. Free Radic Biol Med 48: 120-127, 2010.

26. Zhang M, Wang X, Yao J and Qiu Z: Long non-coding RNA NEAT1 inhibits oxidative stress-induced vascular endothelial cell injury by activating the miR-181d-5p/CDKN3 axis. Artif Cells Nanomed Biotechnol 47: 3129-3137, 2019.

27. Nakamura-Utsunomiya A, Tsumura M, Okada S, Kawaguchi H and Kobayashi M: Downregulation of endothelial nitric oxide synthase (eNOS) and endothelin-1 (ET-1) in a co-culture system with human stimulated X-linked CGD neutrophils. PLoS One 15: e0230665, 2020.

28. Walshe TE, Ferguson G, Connell P, O'Brien C and Cahill PA: Pulsatile flow increases the expression of eNOS, ET-1, and prostacyclin in a novel in vitro coculture model of the retinal vasculature. Invest Ophthalmol Vis Sci 46: 375-382, 2005.

29. Castilla-Guerra L, Fernandez-Moreno MD and ColmeneroCamacho MA: Statins in Stroke Prevention: Present and future. Curr Pharm Des 22: 4638-4644, 2016.

30. Koushki K, Shahbaz SK, Mashayekhi K, Sadeghi M, Zayeri ZD, Taba MY, Banach M, Al-Rasadi K, Johnston TP and Sahebkar A: Anti-inflammatory action of statins in cardiovascular disease: The role of inflammasome and toll-like receptor pathways. Clin Rev Allergy Immunol 60: 175-199, 2021.

31. Mo S, Qian Y, Zhang W, Qian L, Wang Y, Cailin G and Ding H: Mitogen-activated protein kinase action in plant response to high-temperature stress: A mini review. Protoplasma 258: 477-482, 2021.

32. Kim M, Jeong S, Lim CW and Lee SC: Mitogen-activated protein kinase CaDIMK1 functions as a positive regulator of drought stress response and abscisic acid signaling in capsicum annuum. Front Plant Sci 12: 646707, 2021.

33. Lochhead PA, Clark J, Wang LZ, Gilmour L, Squires M, Gilley R, Foxton C, Newell DR, Wedge SR and Cook SJ: Tumor cells with KRAS or BRAF mutations or ERK5/MAPK7 amplification are not addicted to ERK5 activity for cell proliferation. Cell Cycle 15: 506-518, 2016.

34. Gavine PR, Wang M, Yu D, Hu E, Huang C, Xia J, Su X, Fan J, Zhang $\mathrm{T}$, Ye Q, et al: Identification and validation of dysregulated MAPK7 (ERK5) as a novel oncogenic target in squamous cell lung and esophageal carcinoma. BMC Cancer 15: 454, 2015.

35. Nithianandarajah-Jones GN, Wilm B, Goldring CE, Müller J and Cross MJ: ERK5: structure, regulation and function. Cell Signal 24: 2187-2196, 2012.

This work is licensed under a Creative Commons Attribution-NonCommercial-NoDerivatives 4.0 International (CC BY-NC-ND 4.0) License. 